\title{
Recent Advances in the LEWICE Icing Model
}

\author{
William Wright, Vantage Group, LLC \\ Gene Addy, NASA Glenn \\ Peter Struk, NASA Glenn
}

Tadas Bartkus, Ohio Aerospace Institute

Glenn Research Center 


\section{Outline}

- Thermal Model Modifications

- Thermal Model Validation

- Comparison with Thermal Scaling Data

- Conclusions from Thermal Analysis

- Mixed Phase Additions

- Mixed Phase Calibration

- Comparison with RatFac Data

- Conclusions From Ice Crystal Analysis 


\section{Thermal Model Additions}

- Myers Water Film Model

$$
\frac{\partial h}{\partial t}+\frac{\partial}{\partial x}\left[\frac{h^{3}}{3 \mu_{w}}\left(\sigma \frac{\partial^{3} h}{\partial x^{3}}+G_{3} \frac{\partial h}{\partial x}-G_{1}\right)+\tau_{w} \frac{h^{2}}{2 \mu_{w}}\right]=\frac{\rho_{a}}{\rho_{w}} \beta V_{\infty}
$$

- Surface Water Shedding Model (calibrated)

$$
\frac{\dot{m}_{\text {shed }}}{\dot{m}_{\text {runback, in }}}=\frac{W e-W e_{c}}{W e} \quad W e=\frac{\rho_{a} V_{a}^{2} x_{k}}{\sigma} \quad W e_{c}=200+5 * 10^{5} x_{k}
$$

- Enhanced Evaporation

- Chilton-Colburn analogy underestimates evaporation rate by $30 \%$

\section{Glenn Research Center}




\section{Process for Comparison}

- Determine Internal Heat Transfer Coefficient from Dry Cases

- All Cases Use Same Coefficients

$$
N u=0.004 \operatorname{Re}^{1 / 3}\left(\frac{z_{n}}{d_{h}}\right)^{-0.22}\left(\frac{x}{d_{h}}\right)^{-0.38}
$$

- External Heat Transfer Coefficient is Forced Laminar Where There is No Ice

- Run All Dry Cases To Ensure Correlation Matches

- Run Wet Cases for Validation

Glenn Research Center 


\section{Conditions Used For Thermal Comparison}

\begin{tabular}{|l|l|l|l|l|l|l|l|}
\hline Case & $\mathbf{P ( P a )}$ & $\mathbf{V ( m / s )}$ & $\mathbf{T ( K )}$ & LWC & MVD & AOA & $\mathbf{t}(\mathbf{m i n})$ \\
\hline Warm Hold(Ref) & 57295 & 92.7 & 264.5 & 0.5 & 20 & 0 & 7 \\
\hline Warm Hold(Scale) & 98525 & 54.3 & 266.9 & 0.85 & 27.8 & 0 & 7 \\
\hline Descent(Ref) & 69981 & 92.7 & 253.1 & 0.15 & 20 & 0 & 7 \\
\hline Descent(Scale) & 97422 & 66.8 & 254.9 & 0.21 & 24.5 & 0 & 7 \\
\hline Cold Hold(Ref) & 57295 & 92.7 & 247.4 & 0.15 & 20 & 0 & 10 \\
\hline Cold Hold(Scale) & 98318 & 54.6 & 245.5 & 0.25 & 27.8 & 0 & 10
\end{tabular}

\section{Glenn Research Center}




\section{Warm Hold (Ref) - Dry}

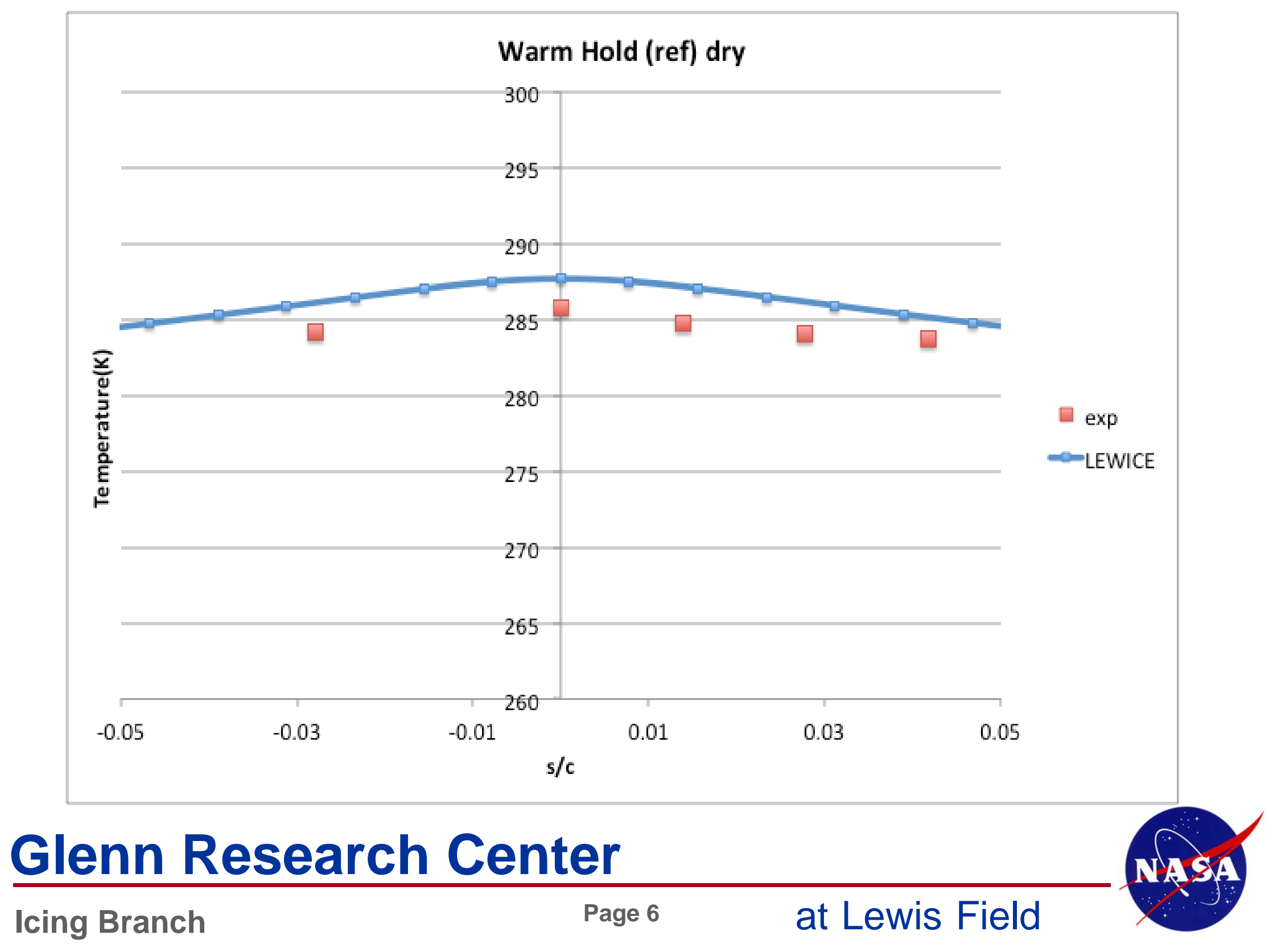




\section{Warm Hold (ref) - Wet}

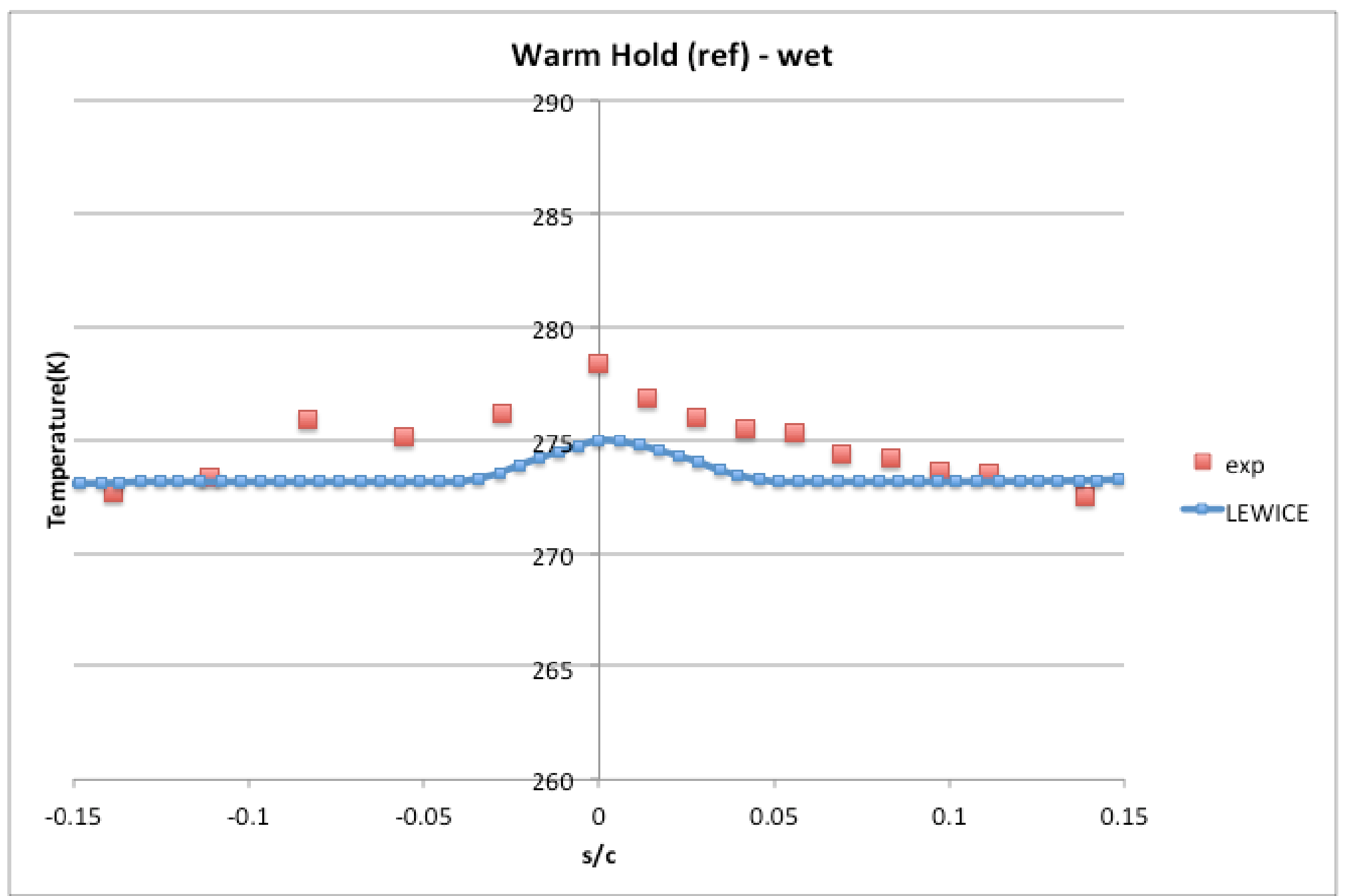

\section{Glenn Research Center}




\section{Warm Hold (Ref) Ice Shape Comparison}

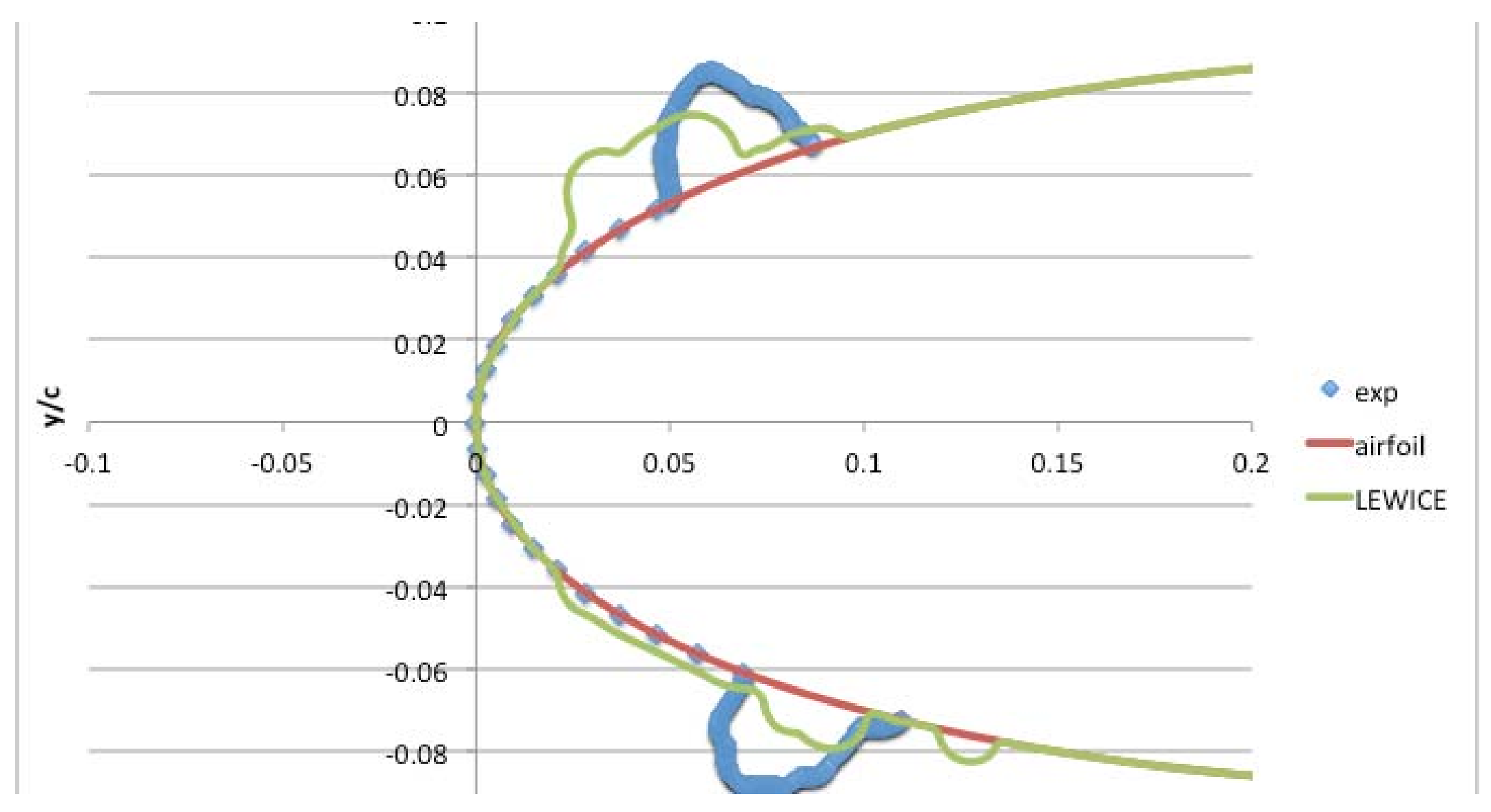

\section{Glenn Research Center}




\section{Warm Hold (Re Scale) - Dry}

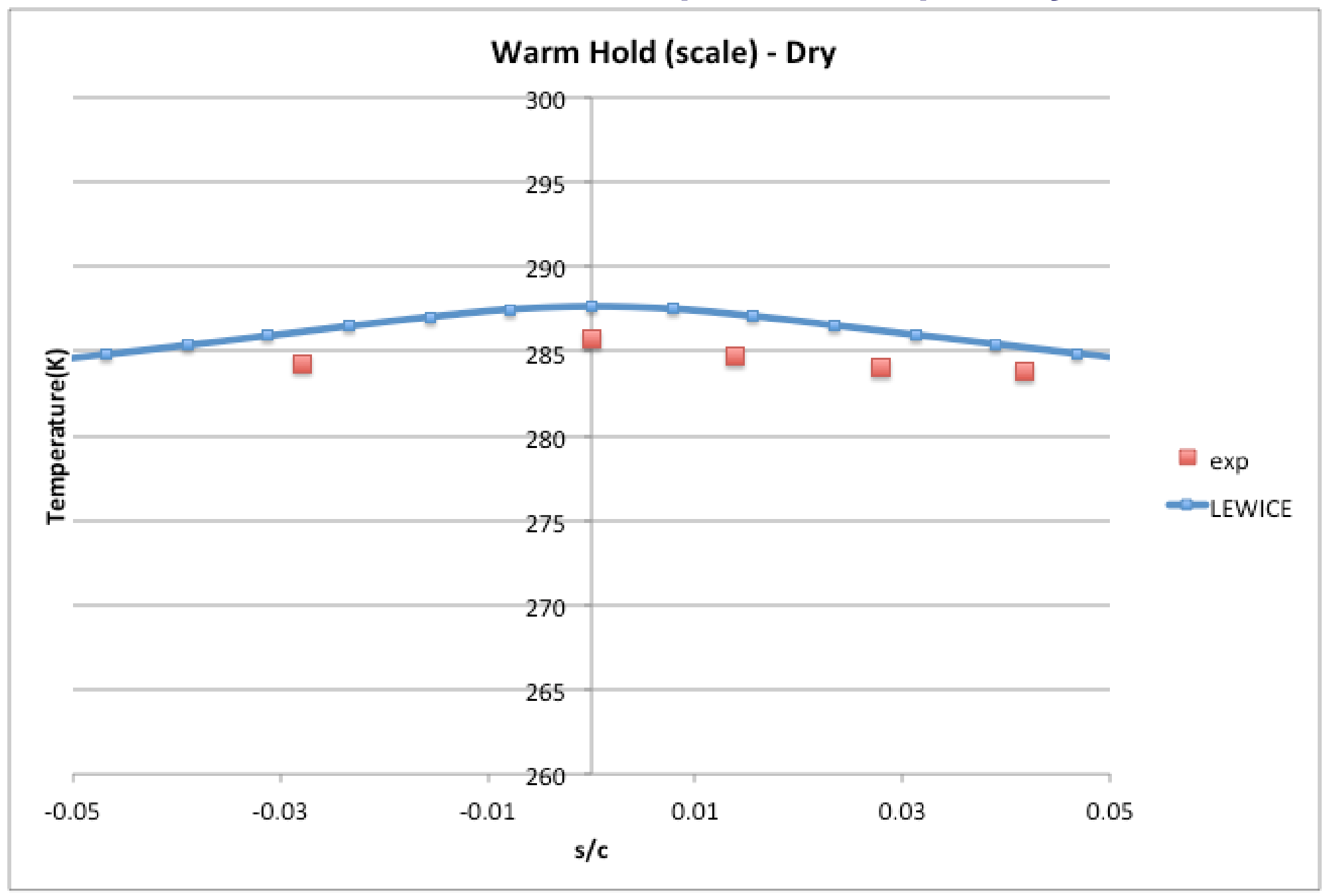

\section{Glenn Research Center}




\section{Warm Hold (Re Scale) - Wet}

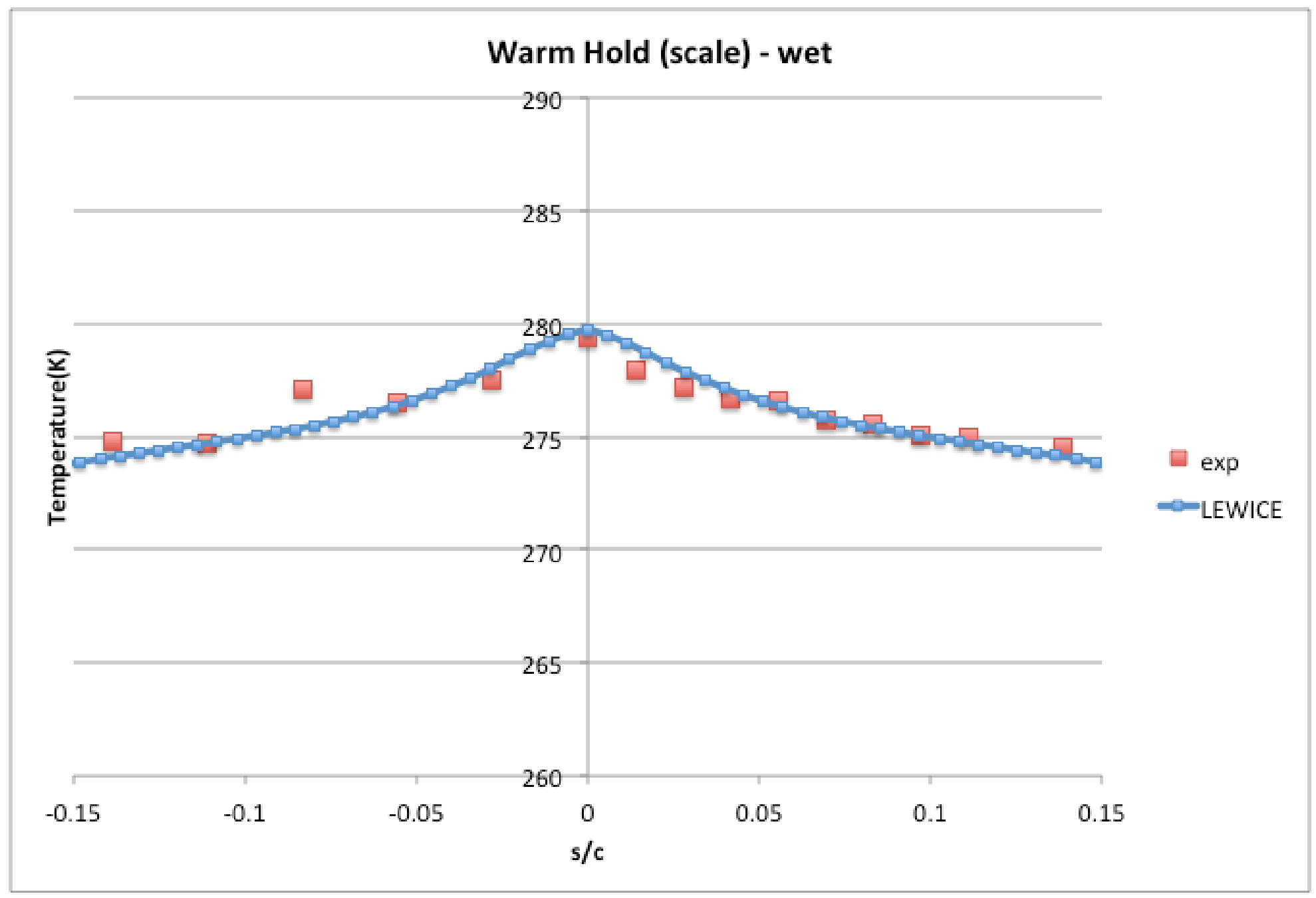

\section{Glenn Research Center}




\section{Warm Hold (Re Scale) Ice Shape}

- No Ice from Experiment nor from LEWICE

\section{Glenn Research Center}




\section{Descent (Ref)}

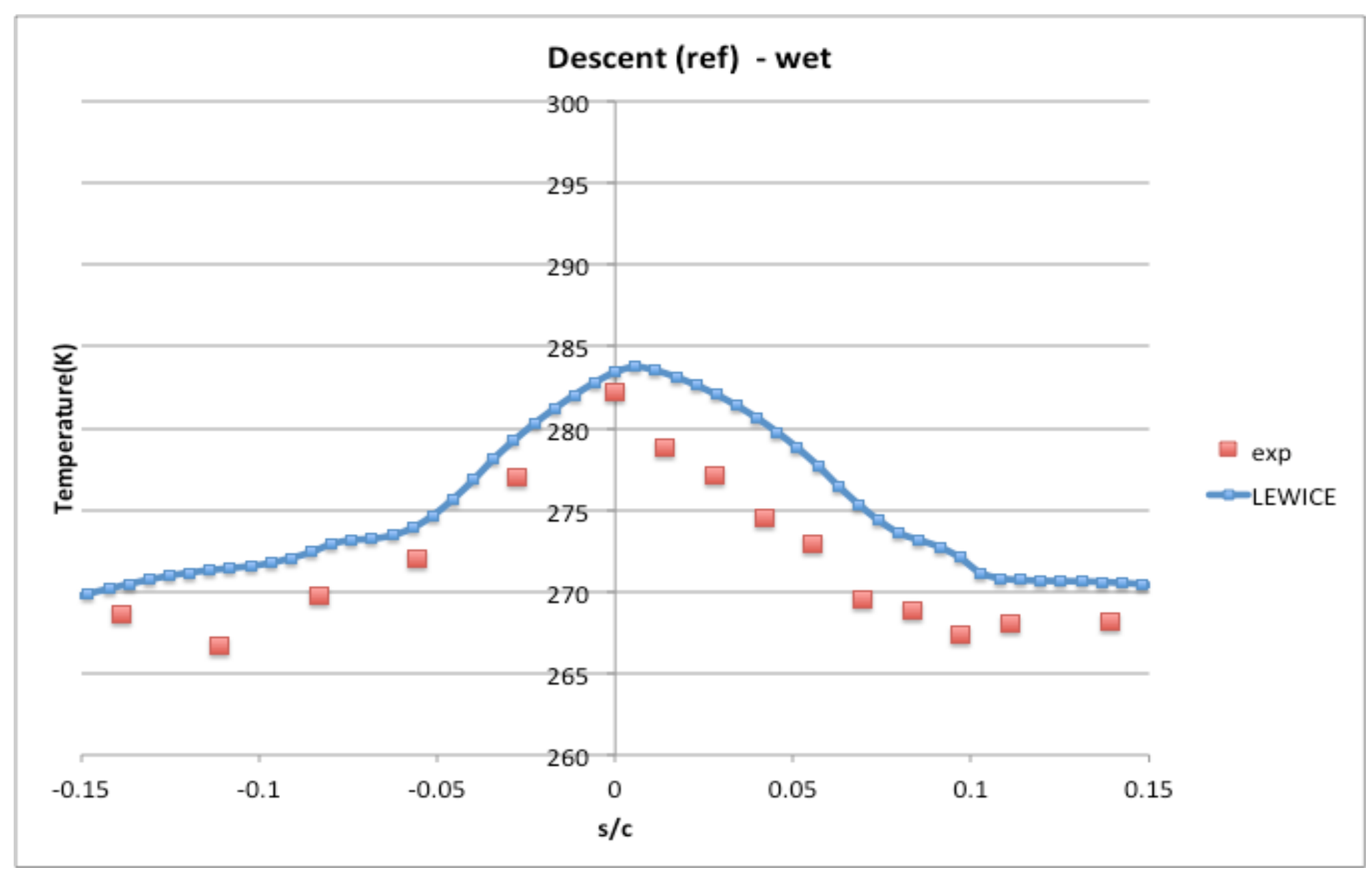

Glenn Research Center

Icing Branch

Page 12

at Lewis Field 


\section{Descent (Ref)}

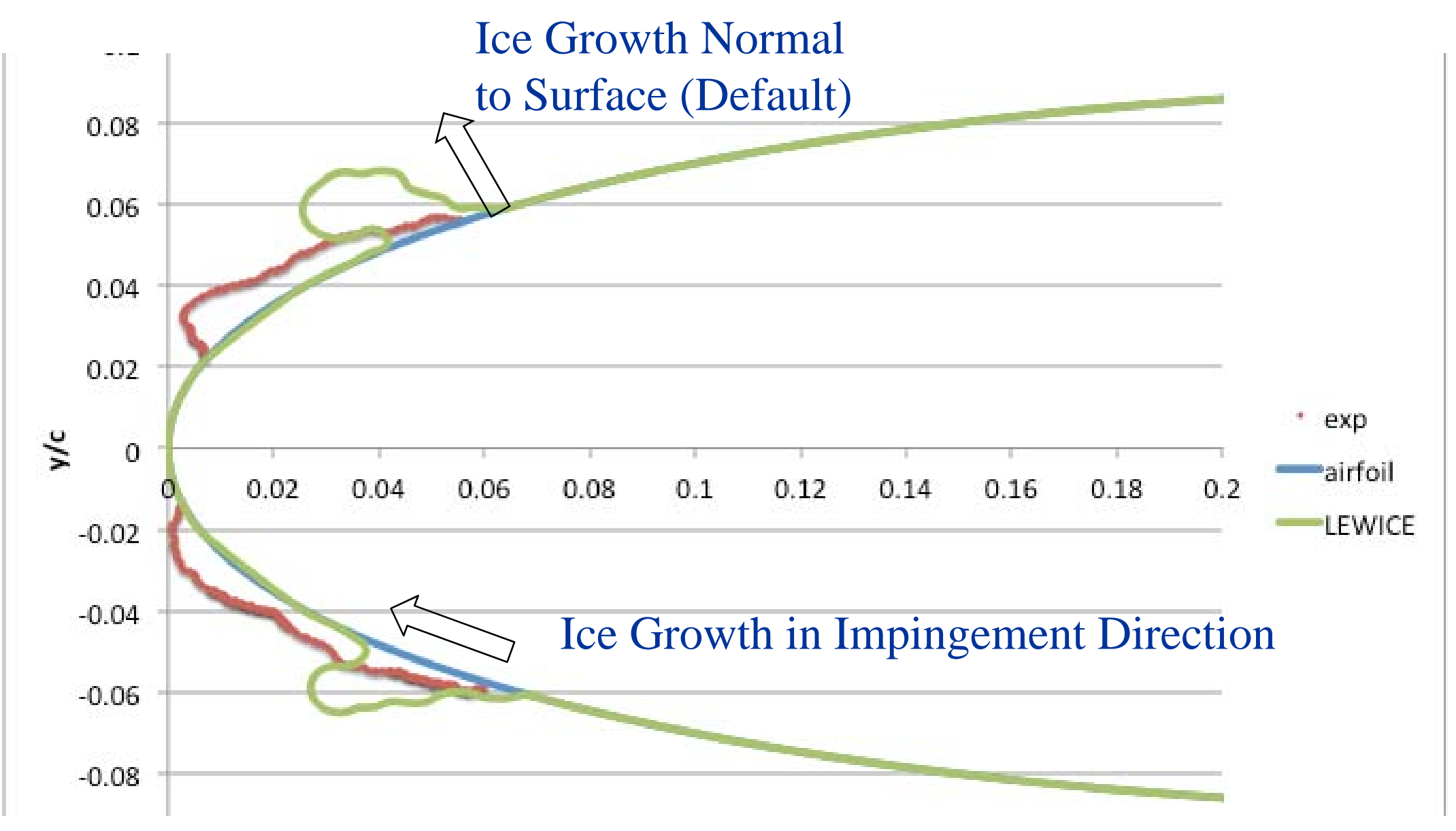

\section{Glenn Research Center}




\section{Descent (Re Scale)}

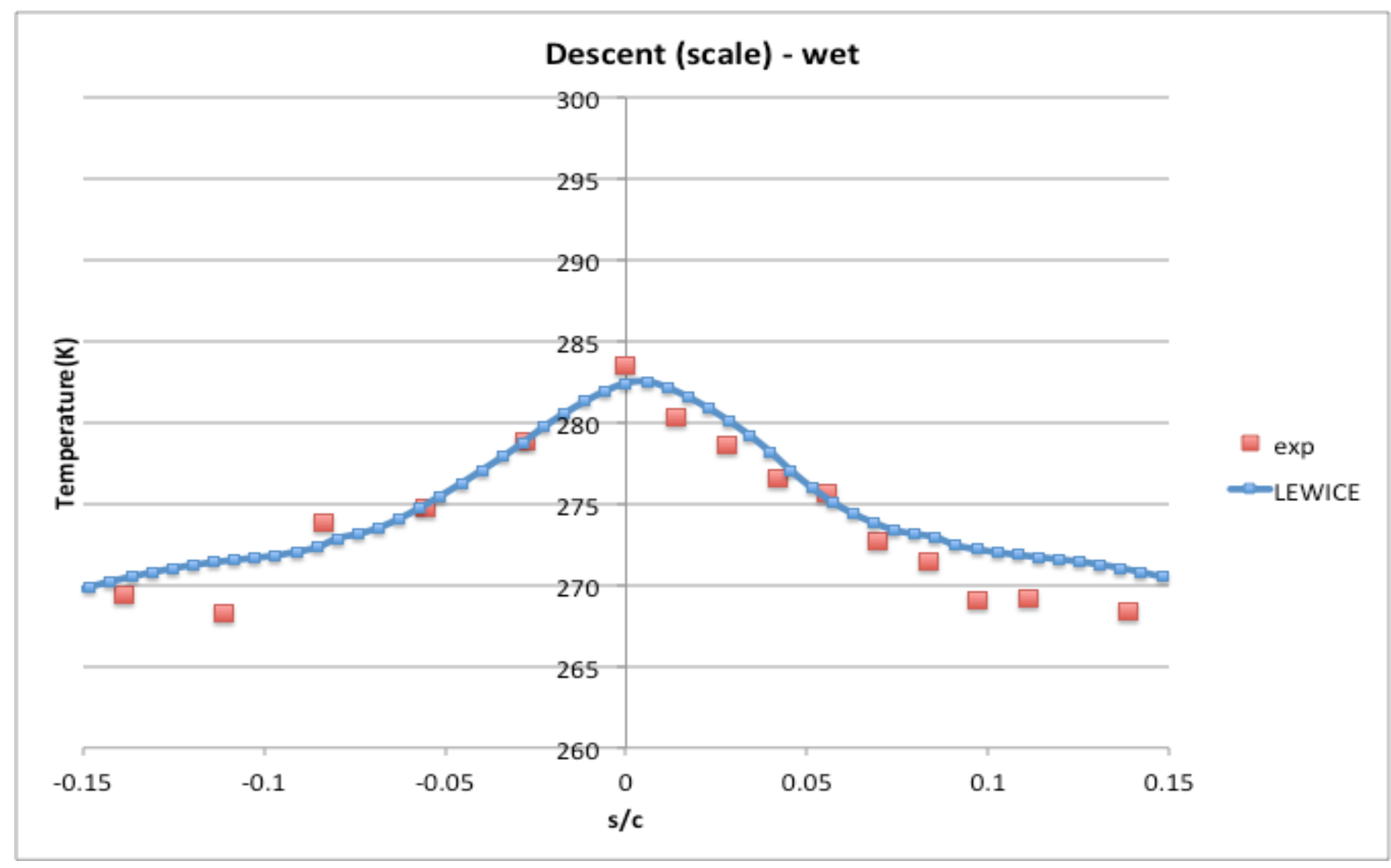

\section{Glenn Research Center}




\section{Descent (Re Scale)}

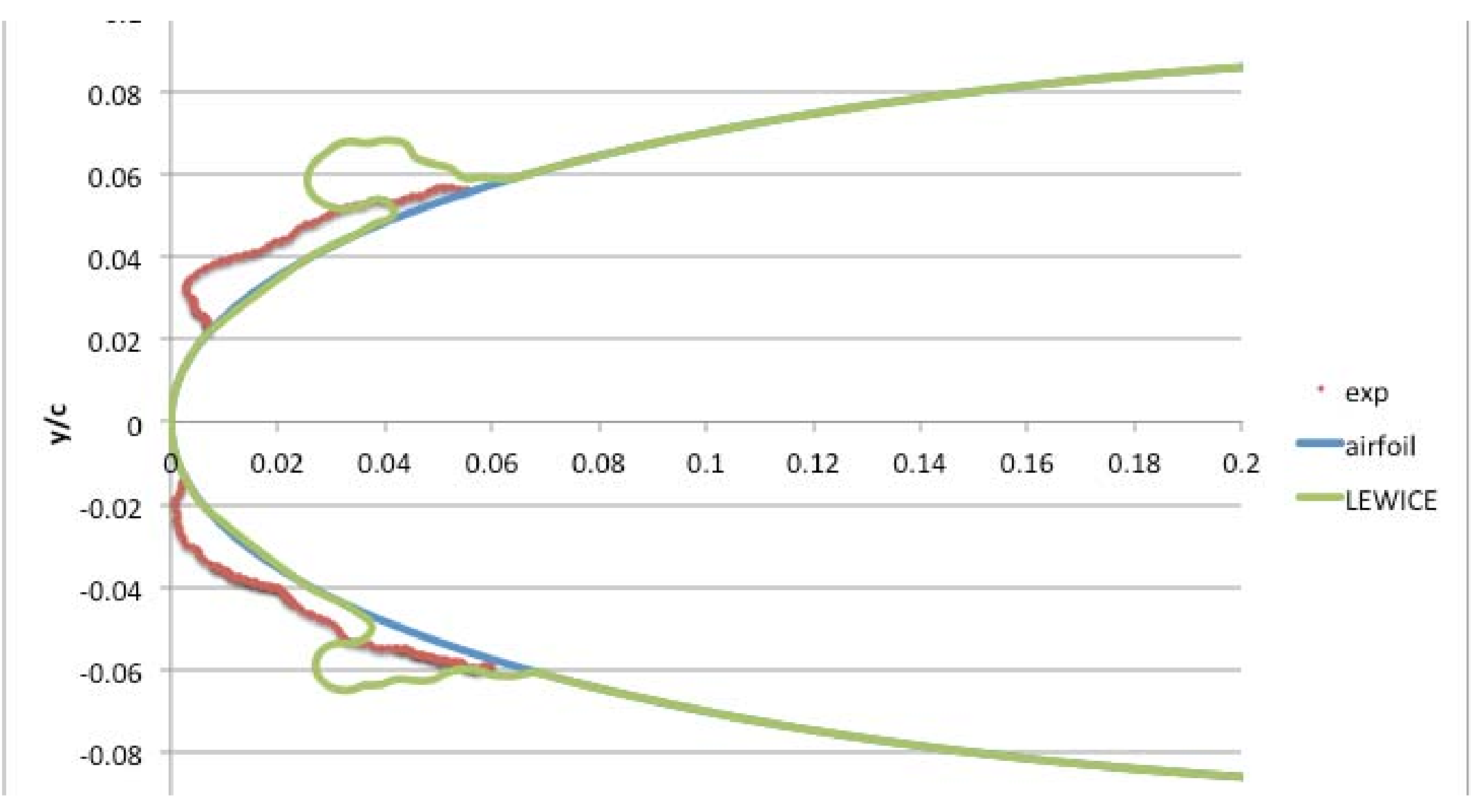

\section{Glenn Research Center}




\section{Cold Hold (Ref) - wet}

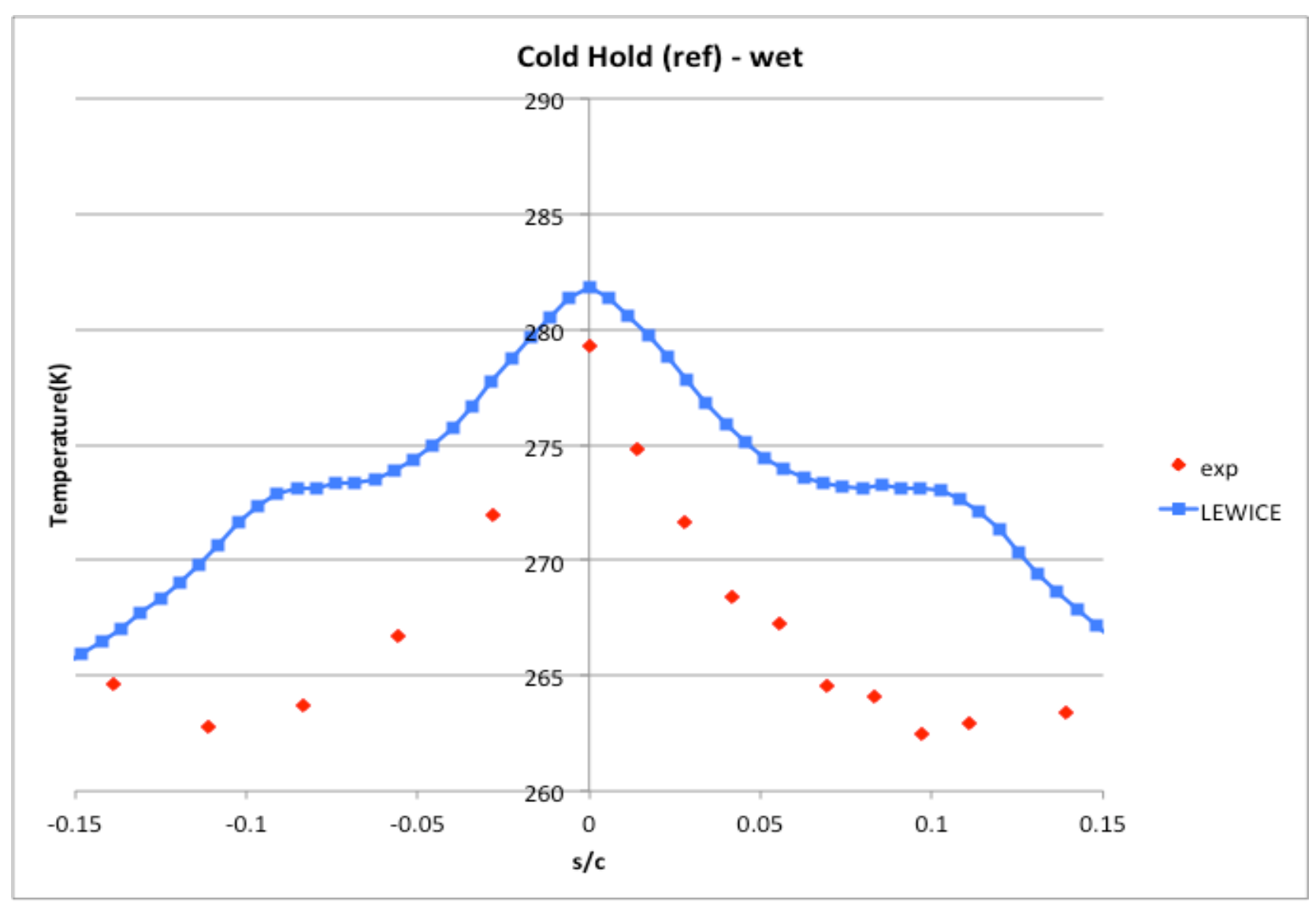

\section{Glenn Research Center}




\section{Cold Hold (Ref)}

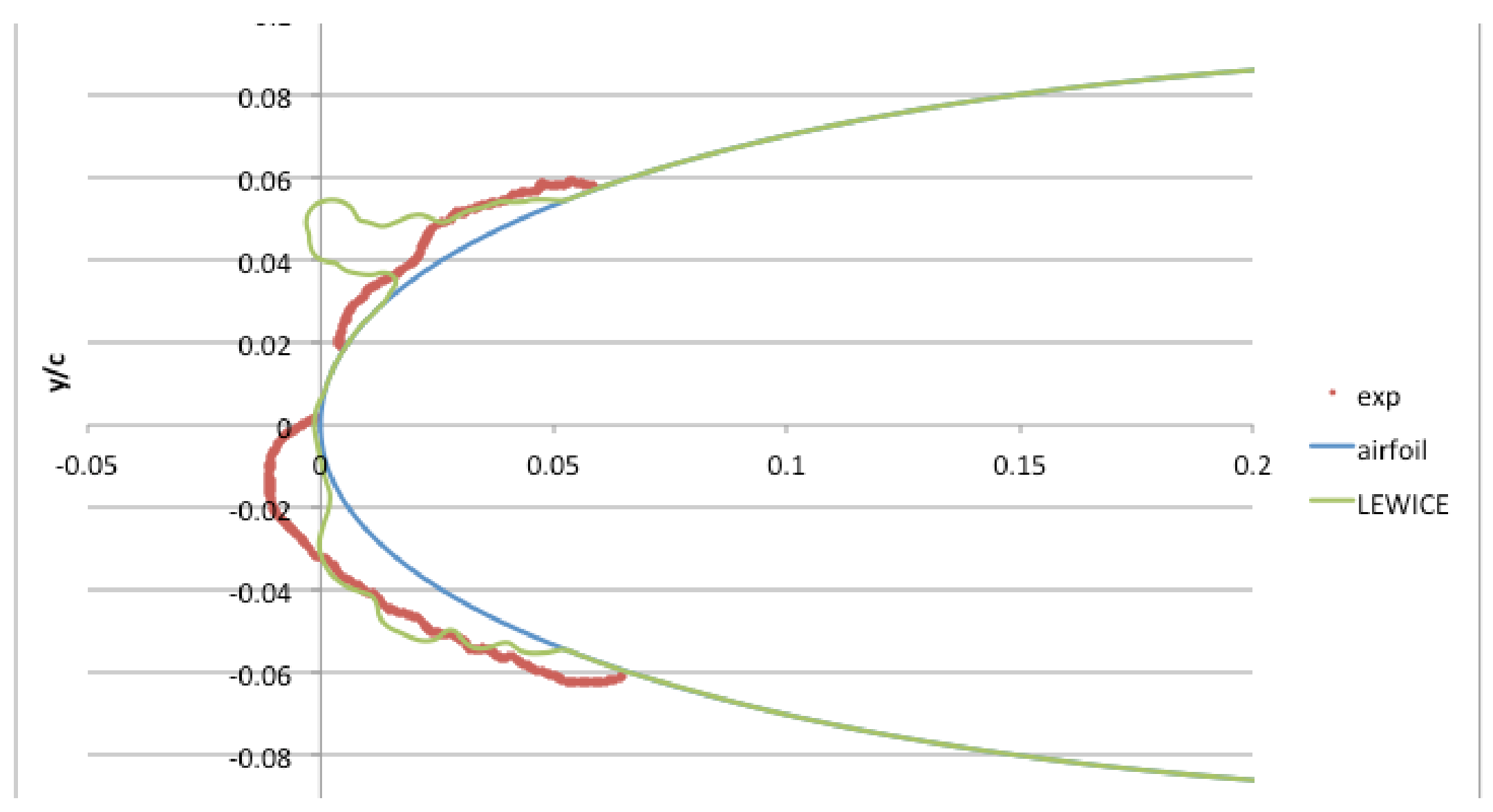

\section{Glenn Research Center}




\section{Cold Hold (Re Scale) - wet}

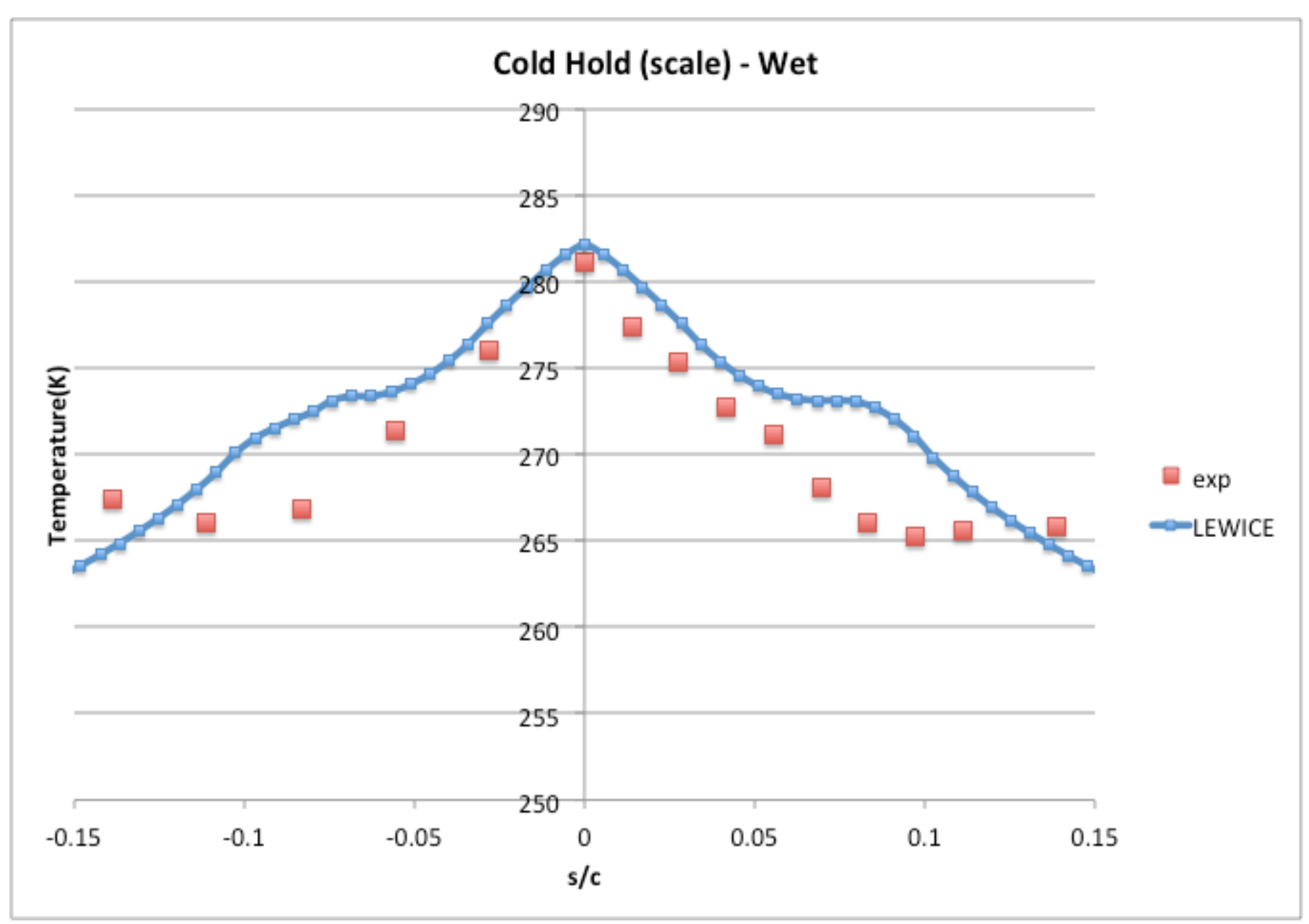

\section{Glenn Research Center}




\section{Cold Hold (Re Scale)}

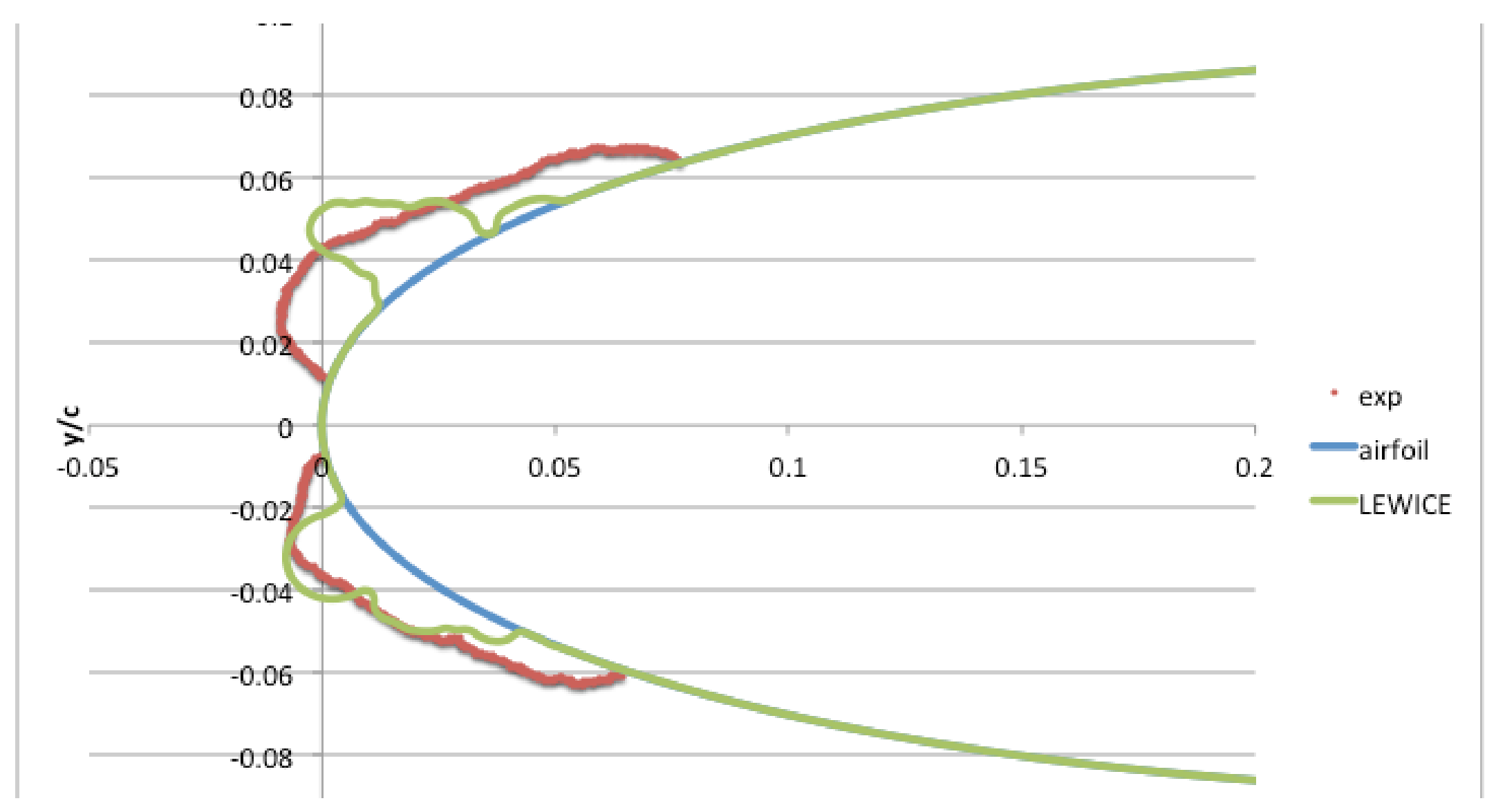

\section{Glenn Research Center}




\section{Observations from Thermal Analysis}

- Temperature Prediction is Very Good to Excellent for Most Cases

- Warm Hold Cases Show Predicted Runback Ice Forward of Experiment

- Peak Ice Thickness Higher for LEWICE

- Descent and Cold Show Predicted Runback Ice Forms Slightly Behind Experiment

- Peak Thickness Higher for LEWICE, Especially Upper Surface

- Ice in Experiment Grows Toward Leading Edge While LEWICE always grows Ice Normal to Surface

- Further Refinement of Runback Model May Be Necessary

- External Heat Transfer Coefficients for Residual Ice Shapes Need to Be Separately Validated

Glenn Research Center 


\section{Ice Breakup Model}

- Breakup Threshold (Hauk)

$$
V_{i m p} \geq \frac{0.45}{\sin \alpha \sqrt{d}}
$$

- Sticking Efficiency (Currie)

$$
\begin{aligned}
\frac{m_{b}}{m_{o}} & =\left(1-\xi \cos \left(\alpha_{i m p}\right)\right)\left(0.57+7.5 * 10^{-4}\left[V_{i m p} \cos \left(\alpha_{i m p}\right)\right]^{1.5}\right) \\
\xi & =-0.1425+47.292 T W C-1979.167 T W C^{2} \\
& - \text { For TWC }<0.12 \mathrm{~kg} / \mathrm{m}^{3} \text { and } \xi=0.14 \text { for TWC }>0.12
\end{aligned}
$$

\section{Glenn Research Center}




\section{Conditions for Ice Crystal Comparison}

\begin{tabular}{|l|l|l|l|l|l|l|l|l|l|}
\hline Airfoil & Scan\# & $\mathbf{P}$ & $\mathbf{V}$ & $\mathbf{T}$ & $\mathbf{S h}$ & LWC & IWC & AOA & $\mathbf{t}(\mathbf{m})$ \\
\hline Wedge & 889 & 6.5 & 87.4 & 12.7 & 8.3 & 1.4 & 4.4 & -6 & 3 \\
\hline Wedge & 996 & 10 & 83.9 & 4.3 & 5.6 & 1.3 & 6.9 & -6 & 3.5 \\
\hline $\begin{array}{l}\text { Wedge } \\
\text { NACA }\end{array}$ & 1003 & 10 & 84.1 & 3.8 & 5.2 & 1.9 & 7.3 & -6 & 3.5 \\
\hline 0012 & 796 & 6.5 & 86.2 & 7.2 & 5.9 & 0.6 & 4.9 & 0 & 3 \\
\hline
\end{tabular}

\section{Glenn Research Center}




\section{Ice Shape Prediction for Scan 996}
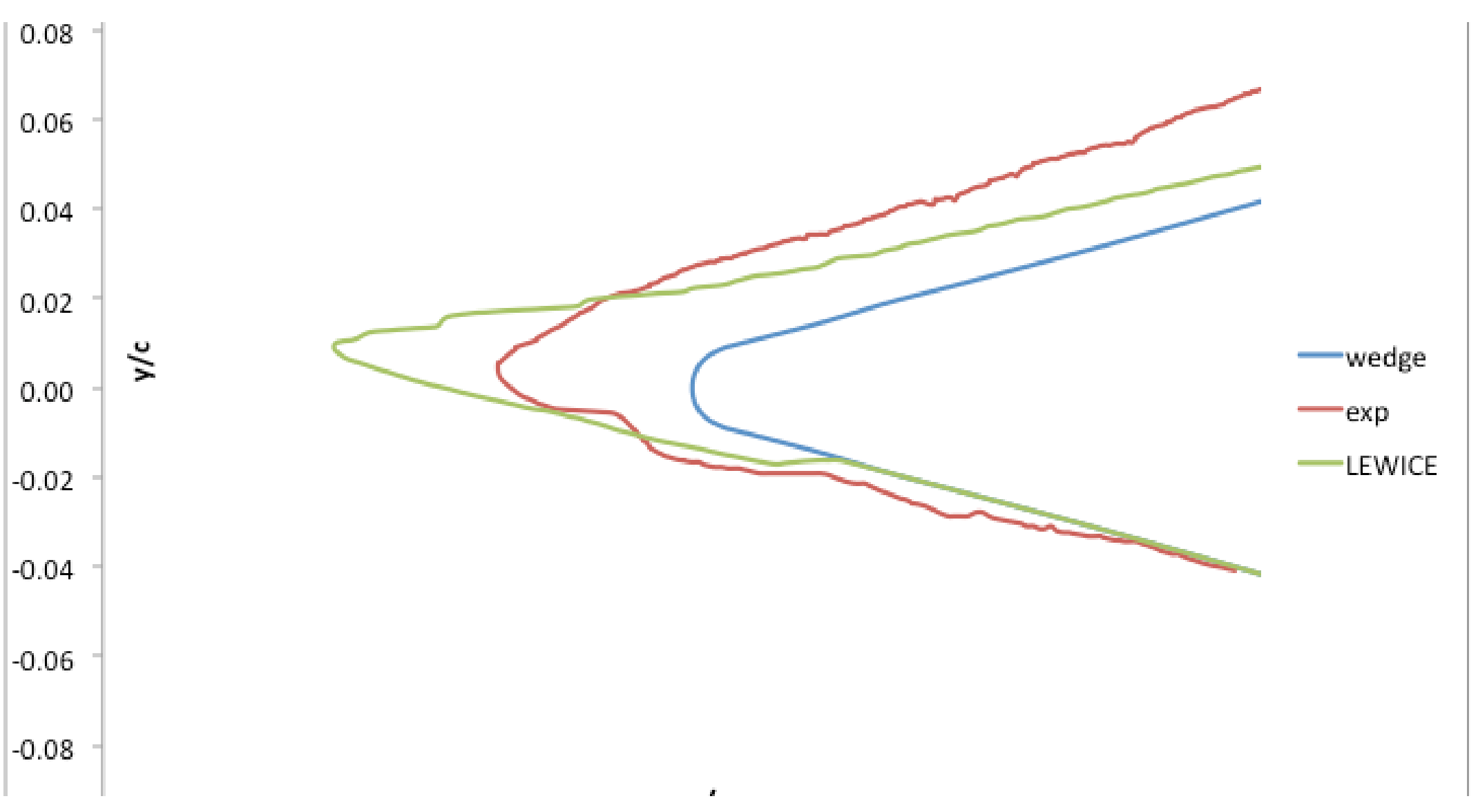

\section{Glenn Research Center}




\section{Ice Shape Prediction for Scan 1003}

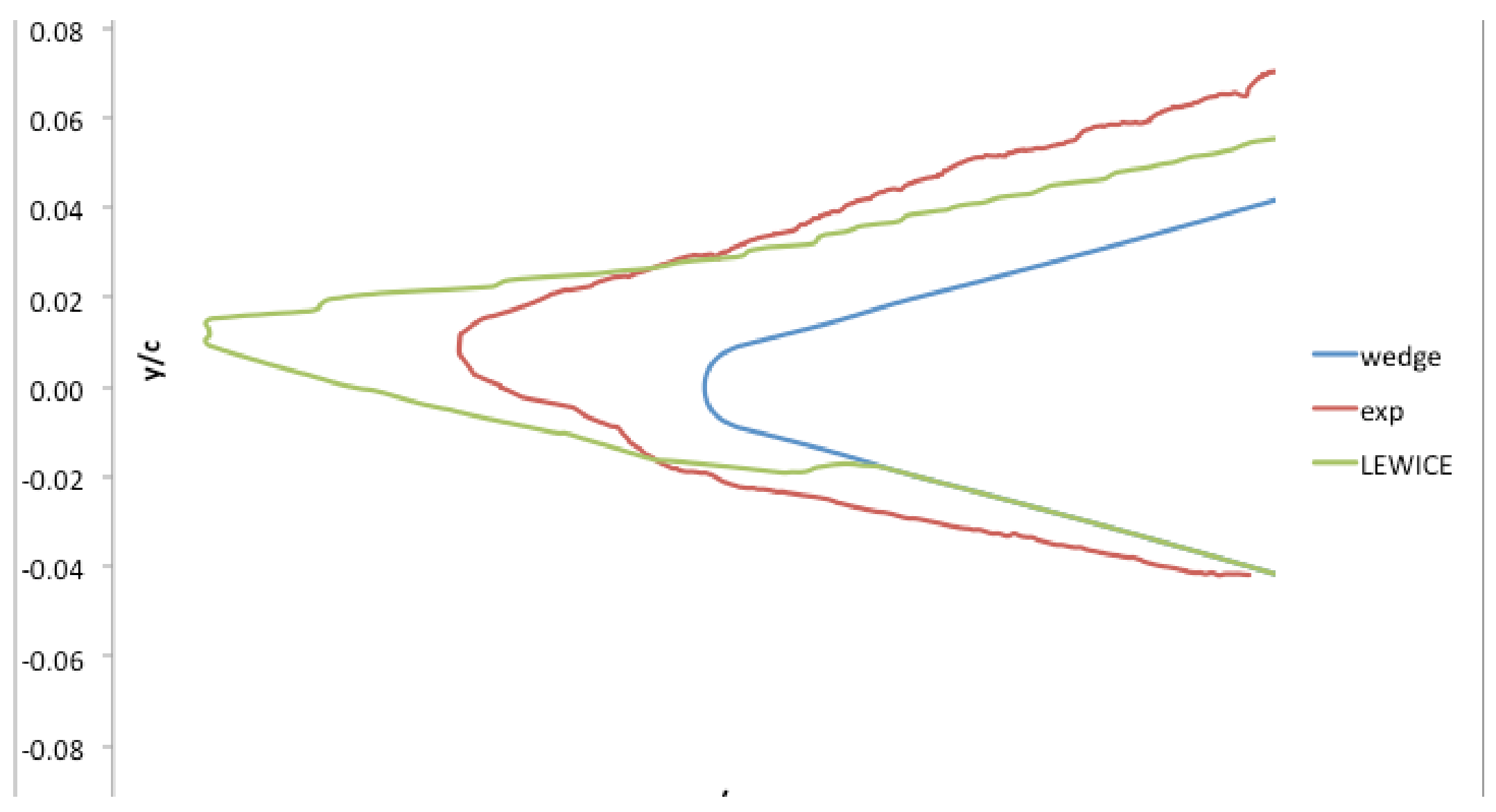

\section{Glenn Research Center}




\section{Sticking Efficiency on Wedge at Various Particle Sizes}

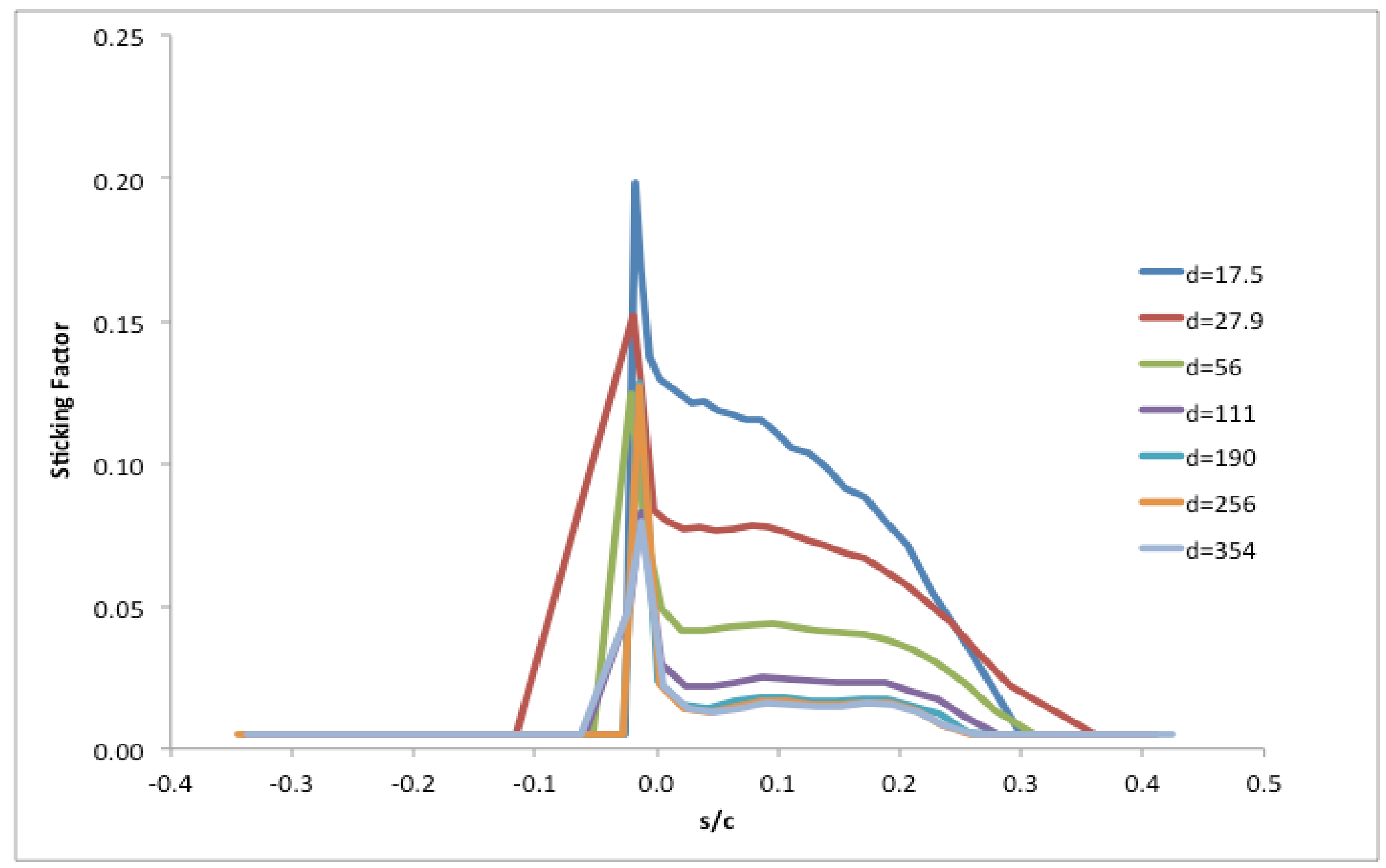

Glenn Research Center 


\section{Ice Thickness Prediction for Scan 796 (NACA0012)}

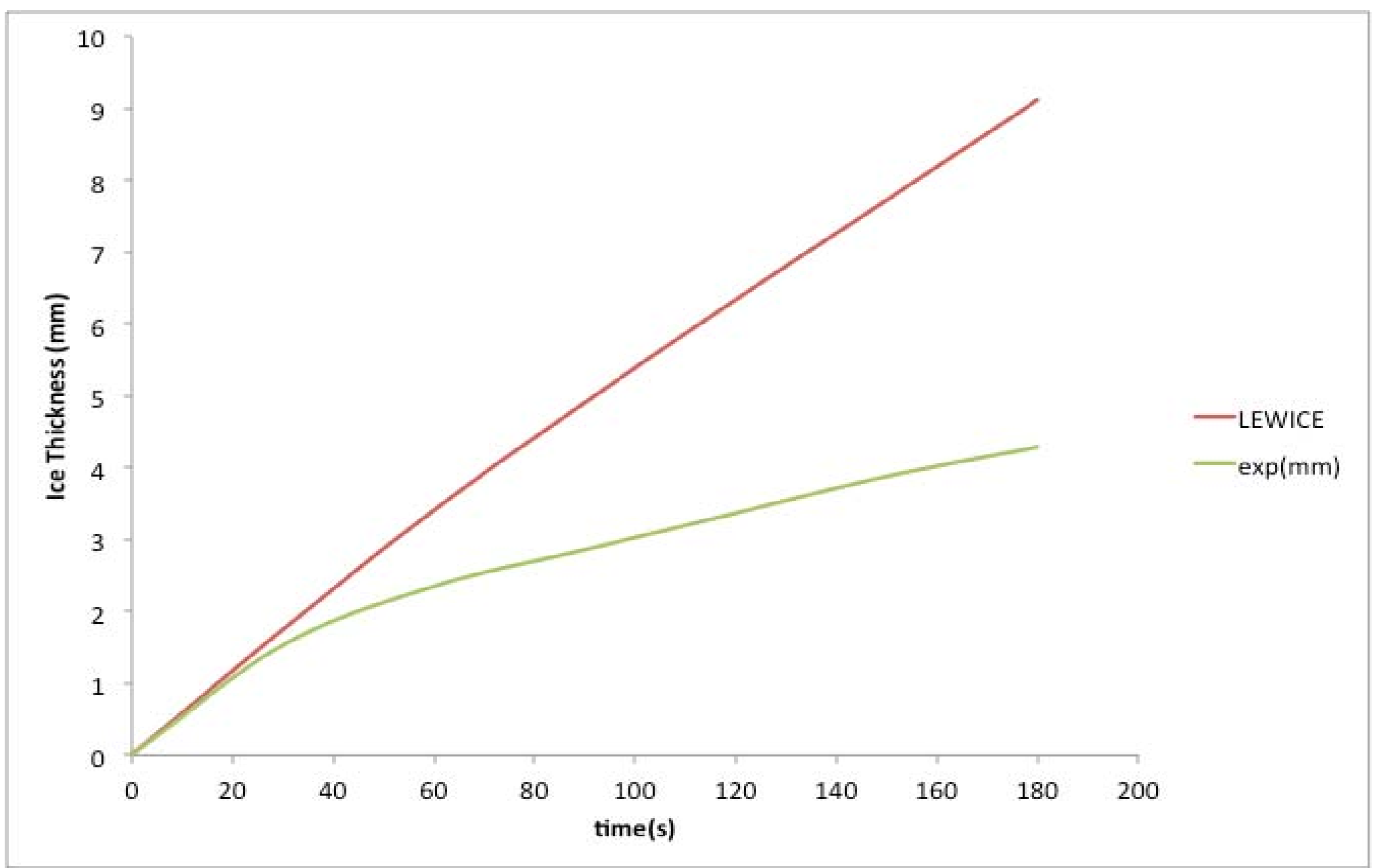

Glenn Research Center 


\section{Observations from Ice Crystal Comparison}

- Peak Thickness is Over Predicted by LEWICE while Extent is Under Predicted

- Additional Erosion Effects may be Needed

- Improved Model for Reimpingement of Ice Crystals

- Additional Data is Needed to Complete Model 